OPEN ACCESS

Edited by: Eduard Kerkhoven, Chalmers University of Technology, Sweden

Reviewed by: Mario Andrea Marchisio, Tianjin University, China Cleo Kontoravdi, Imperial College London, United Kingdom

${ }^{*}$ Correspondence:

Hector Garcia Martin hgmartin@lbl.gov

tThese authors have contributed equally to this work

Specialty section:

This article was submitted to

Synthetic Biology

a section of the journal Frontiers in Bioengineering and Biotechnology

Received: 01 October 2020 Accepted: 13 January 2021

Published: 09 February 2021

Citation:

Roy S, Radivojevic T, Forrer M, Marti JM, Jonnalagadda $V$, Backman T, Morrell W, Plahar H, Kim J, Hillson N and Garcia Martin H (2021) Multiomics Data Collection, Visualization, and Utilization for Guiding Metabolic Engineering. Front. Bioeng. Biotechnol. 9:612893. doi: 10.3389/fbioe.2021.612893

\section{Multiomics Data Collection, Visualization, and Utilization for Guiding Metabolic Engineering}

\author{
Somtirtha Roy ${ }^{1,2 t}$, Tijana Radivojevic ${ }^{1,2,3 \dagger}$, Mark Forrer ${ }^{2,3,4}$, Jose Manuel Marti ${ }^{1,2,3}$, \\ Vamshi Jonnalagadda ${ }^{1,2}$, Tyler Backman ${ }^{1,3}$, William Morrell ${ }^{2,3,4}$, Hector Plahar ${ }^{1,2}$, \\ Joonhoon Kim ${ }^{3,5}$, Nathan Hillson ${ }^{1,2,3}$ and Hector Garcia Martin 1,2,3,6* \\ ${ }^{1}$ Lawrence Berkeley National Laboratory, Biological Systems and Engineering Division, Berkeley, CA, United States, \\ ${ }^{2}$ Department of Energy, Agile BioFoundry, Emeryville, CA, United States, ${ }^{3}$ Joint BioEnergy Institute, Emeryville, CA, \\ United States, ${ }^{4}$ Sandia National Laboratories, Biomaterials and Biomanufacturing, Livermore, CA, United States, ${ }^{5}$ Chemical \\ and Biological Processes Development Group, Pacific Northwest National Laboratory, Richland, WA, United States, ${ }^{6}$ BCAM, \\ Basque Center for Applied Mathematics, Bilbao, Spain
}

Biology has changed radically in the past two decades, growing from a purely descriptive science into also a design science. The availability of tools that enable the precise modification of cells, as well as the ability to collect large amounts of multimodal data, open the possibility of sophisticated bioengineering to produce fuels, specialty and commodity chemicals, materials, and other renewable bioproducts. However, despite new tools and exponentially increasing data volumes, synthetic biology cannot yet fulfill its true potential due to our inability to predict the behavior of biological systems. Here, we showcase a set of computational tools that, combined, provide the ability to store, visualize, and leverage multiomics data to predict the outcome of bioengineering efforts. We show how to upload, visualize, and output multiomics data, as well as strain information, into online repositories for several isoprenol-producing strain designs. We then use these data to train machine learning algorithms that recommend new strain designs that are correctly predicted to improve isoprenol production by $23 \%$. This demonstration is done by using synthetic data, as provided by a novel library, that can produce credible multiomics data for testing algorithms and computational tools. In short, this paper provides a step-by-step tutorial to leverage these computational tools to improve production in bioengineered strains.

Keywords: machine learning, flux analysis, metabolic engineering, biofuels, synthetic biology, multiomics analysis

\section{INTRODUCTION}

Synthetic biology represents another step in the development of biology as an engineering discipline. The application of engineering principles such as standardized genetic parts (Canton et al., 2008; Müller and Arndt, 2012) or the application of Design-Build-Test-Learn (DBTL) cycles (Petzold et al., 2015; Nielsen and Keasling, 2016) has transformed genetic and metabolic engineering in significant ways. Armed with this new engineering framework, synthetic biology is creating products to tackle societal problems in ways that only biology can enable. Synthetic biology, for example, is being leveraged to produce renewable biofuels to combat climate change 
(Peralta-Yahya et al., 2012; Beller et al., 2015; Chubukov et al., 2016), improve crop yields (Roell and Zurbriggen, 2020), combat the spread of diseases (Kyrou et al., 2018), synthesize medical drugs (Ajikumar et al., 2010; Paddon and Keasling, 2014), biomaterials (Bryksin et al., 2014), and plant-based foods (Meatfree outsells beef, 2019).

However, the development of synthetic biology is hindered by our inability to predict the results of engineering outcomes. DNA synthesis and CRISPR-based genetic editing (Ma et al., 2012; Doudna and Charpentier, 2014) allow us to produce and change DNA (the working code of the cell) with unparalleled ease, but we can rarely predict how that modified DNA will impact cell behavior (Gardner, 2013). As a consequence, it is not possible to design a cell to fit a desired specification: e.g., have the cell produce $X$ grams of a specified biofuel or an anticancer agent. Hence, metabolic engineering is often mired in trial-anderror approaches that result in very long development times (Hodgman and Jewett, 2012). In this context, machine learning has recently appeared as a powerful tool that can provide the predictive power that bioengineering needs to be effective and impactful (Carbonell et al., 2019; Radivojević et al., 2020; Zhang et al., 2020).

Furthermore, although there is a growing abundance of phenotyping data, the tools to systematically leverage these data to improve predictive power are lacking. For example, transcriptomics data has a doubling time of 7 months (Stephens et al., 2015), and high-throughput techniques for proteomics (Chen et al., 2019) and metabolomics (Fuhrer and Zamboni, 2015) are becoming increasingly available. Often, metabolic engineers struggle to synthesize this data deluge into precise actionable items (e.g., down regulate this gene and knock out this transcription factor) to obtain their desired goal (e.g., increase productivity to commercially viable levels).

Here, we showcase how to combine the following existing tools to leverage omics data and suggest next steps (Figure 1): the Inventory of Composable Elements (ICE), the Experiment Data Depot (EDD), the Automated Recommendation Tool (ART), and Jupyter Notebooks. ICE (Ham et al., 2012) is an open source repository platform for managing information about DNA parts and plasmids, proteins, microbial host strains, and plant seeds. EDD (Morrell et al., 2017) is an open source online repository of experimental data and metadata. ART (Radivojević et al., 2020; Zhang et al., 2020) is a library that leverages machine learning for synthetic biology purposes, providing predictive models and recommendations for the next set of experiments. Jupyter notebooks are interactive documents that contain live code, equations, visualizations, and explanatory text (Project Jupyter | Home $^{1}$; IOS Press Ebooks - Jupyter Notebooks) (Kluyver et al., 2016). When combined, this set of tools can effectively store, visualize, and leverage synthetic biology data to enable predictive bioengineering and effective actionable items for the next DBTL cycle. We will demonstrate this with an example in which we leverage multiomics data to improve the production of isoprenol, a potential biofuel (Kang et al., 2019). This multiomics data set

${ }^{1}$ Project Jupyter | Home. Available online at: https://jupyter.org/ (accessed September 4, 2020).

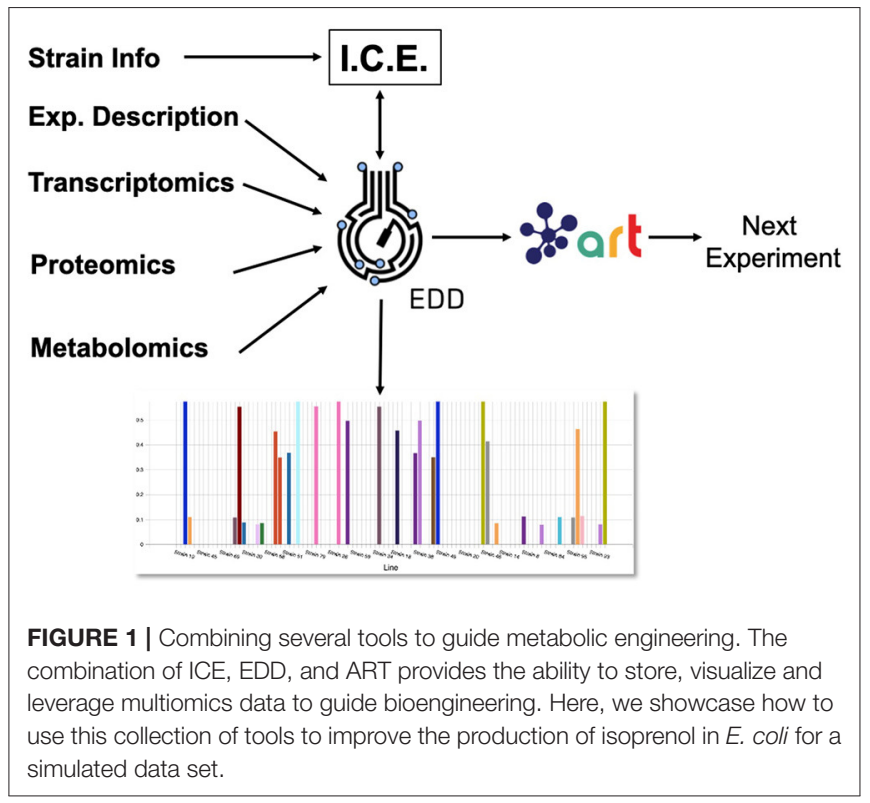

is a synthetic data set (i.e., simulated computationally without experimental work) generated through the new Omics Mock Generator (OMG) library. Synthetic data provide the advantage of easily producing large amounts of multimodal data that would be prohibitively expensive to produce experimentally. In this way, we can concentrate this manuscript on the demonstration of computational tools rather than the details and vagaries of data collection. The OMG library provides an easily accessible source of biologically believable data that can be used to test algorithms and tools systematically. Validation for these tools with experimental data has already been provided elsewhere (e.g., Radivojević et al., 2020; Zhang et al., 2020).

\section{METHODS}

\section{Synthetic Data Generator Library (OMG)}

The Omics Mock Generator (OMG) library is used to provide the synthetic multiomics data needed to test the computational tools described here (Figure 2). Since experimental multiomics data are expensive and non-trivial to produce, OMG provides a quick and easy way to produce large amounts of multiomics data that are based on plausible metabolic assumptions. OMG creates fluxes based on Flux Balance Analysis (FBA) and growth rate maximization (Orth et al., 2010), leveraging COBRApy (Ebrahim et al., 2013). OMG can use any genome-scale model, but in this case we have used the iJO1366 E. coli genome scale model, augmented with an isoprenol pathway obtained from the iMM904 S. cerevisiae model (Notebook A). In order to obtain proteomics data, we assume that the corresponding protein expression and gene transcription are linearly related to the fluxes. We also assume the concentration of metabolites to be loosely related to the fluxes of the reactions that consume or produce them (no fluxes $\rightarrow$ no metabolite): the amount of metabolite present is assumed to be proportional to the sum of absolute fluxes coming in and out of the metabolite. Therefore, although the data provided by OMG is not real, it is more realistic 


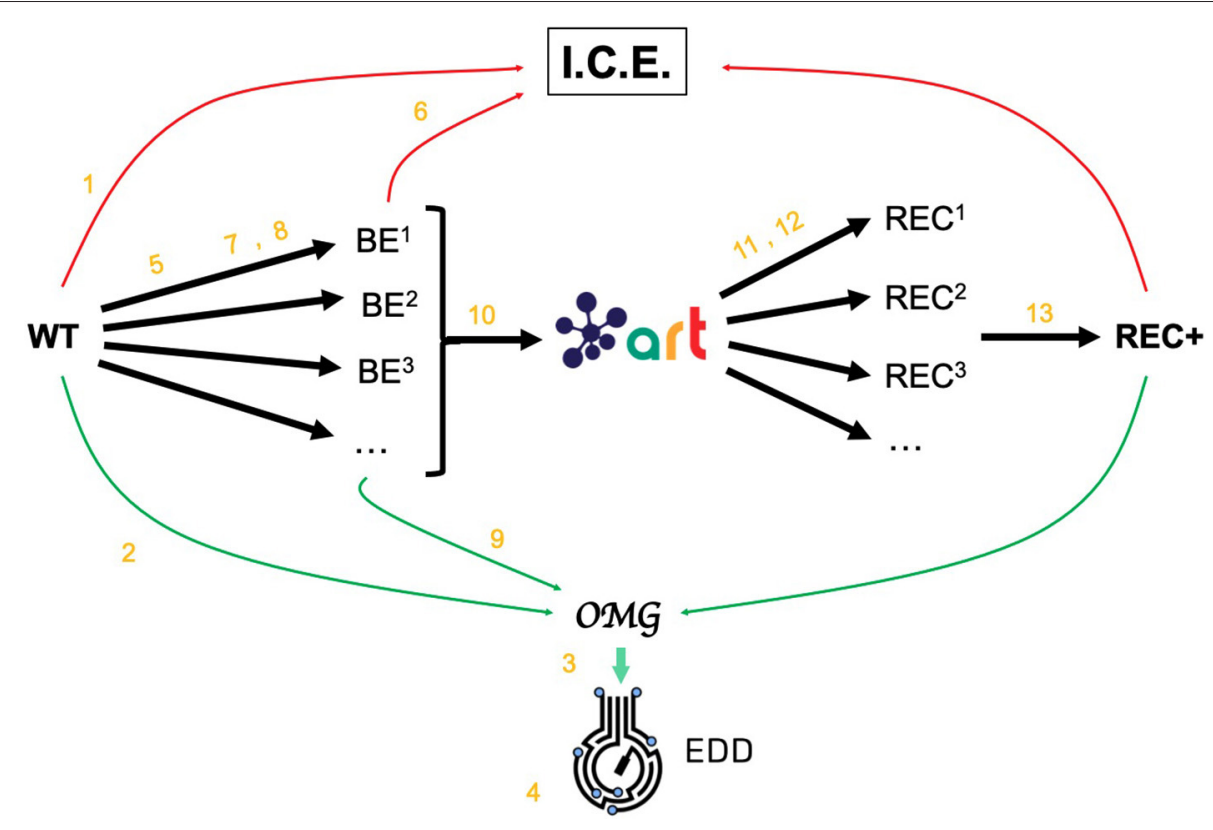

FIGURE 2 | Demonstrating ICE, EDD, and ART using synthetic data. For the purposes of the demonstration of how ICE, EDD, and ART work together, we use a synthetic data set of multiomics data (transcriptomics, proteomics, metabolomics, fluxomics) for several time points created by the Omics Mock Generator (OMG) library (see Methods section). We start with a base strain (wild type, or WT) that is bioengineered according to several designs (i.e., knockout malate dehydrogenase, overexpress citrate synthase) suggested by ART. The results are 95 bioengineered strains (BE ${ }^{1}, \mathrm{BE}^{2}$... etc.) for which experimental data (isoprenol production levels) are simulated through OMG and stored in EDD and ICE. These data are then leveraged by ART to recommend, using machine learning, new designs that are expected to improve isoprenol production $\left(\mathrm{REC}^{1}, \mathrm{REC}^{2}, \ldots\right)$. These recommendations and production predictions are compared with the ground truth provided by OMG. Each of these steps (in orange) is demonstrated through screencasts and Jupyter notebooks (Table 1).

than randomly generated data, providing a useful resource to test the scaling of algorithms and computational tools.

The data generated by OMG was used in this manuscript to test EDD input, output, and visualization, and to provide training data for ART (Figure 2). This tool can be a very useful resource for the rapid prototyping of new tools and algorithms.

\section{Generating Flux Time Series Data}

Fluxes describe the rates of metabolic reactions in a given organism, and can be easily generated through FBA. FBA assumes that the organism is under selective pressure to increase its growth rate (Orth et al., 2010; Lewis et al., 2012), hence searching for the fluxes that optimize it. FBA relies on genomescale models, which provide a comprehensive description of all known genetically encoded metabolic reactions (Thiele and Palsson, 2010). FBA produces fluxes by solving through Linear Programming (LP) the following optimization problem:

$$
\begin{array}{r}
\text { Maximize } V_{\text {biomass }} \text { subject to: } \\
\Sigma_{j} S_{i j} V_{j}=0 \\
l b_{j} \leq V_{j} \leq u b_{j}
\end{array}
$$

where $S$ is the stoichiometry matrix of size $m^{*} n$ (number of metabolites*number of reactions in the model), $V_{j}$ is the flux for reaction $j$ within the model $(j=1, \ldots, n)$. The lower bound $\left(l b_{j}\right)$ and upper bound $\left(u b_{j}\right)$ provide the minimum and maximum for each reaction. For example, if the input carbon source is glucose and we know that the input in a given time lapse is $-15 \mathrm{mmol} / \mathrm{gdw} / \mathrm{h}$, the upper bound and lower bound for the exchange reaction corresponding to glucose are set to this value: $-15<\mathrm{V}_{\mathrm{EX} \text { glc }}$ $<-15$. The solution to this optimization problem provides the fluxes that maximize growth rate, and that will be used later on to obtain transcriptomics, proteomics and metabolomics data. However, one must be aware that this optimization problem is underdetermined, and there are multiple solutions that satisfy exchange flux constraints.

We create time series of fluxes by doing a batch simulation based on FBA (see OMG library and Notebook A). We assume a given concentration for extracellular metabolites (e.g., $22 \mathrm{mM}$ of glucose, or $18 \mathrm{mM}$ of ammonium) and, for each time point, we run FBA for the model and update the extracellular metabolite concentration based on the exchange fluxes coming from the simulation (see Notebook A). For example, if an exchange flux of V_EX_glc_D $=-15 \mathrm{mmol} / \mathrm{gdw} / \mathrm{hr}$ is obtained for the model, the corresponding glucose concentration is adjusted as follows:

$$
\left[g l c \_D\right]_{\text {new }}=\left[g l c \_D\right]_{\text {old }}-15 \mathrm{mmol} / \mathrm{gdw} / \mathrm{hr} \cdot \Delta t \cdot[\text { cell }]
$$

Where $\left[g l c \_D\right]_{\text {old }}$ is the old glucose concentration, $\left[g l c \_D\right]_{n e w}$ is the updated concentration, $\Delta t$ is the time change and [cell] is the cell concentration. In practice, we assume that the cell density increase is better described by an exponential than a 
linear relationship, and $\Delta t \cdot[\mathrm{cell}]$ is substituted by $\Delta[\mathrm{cell}] / \mu$, where $\mu$ is the growth rate: $\mu=1 /[$ cell $] \cdot \Delta[$ cell $] / \Delta t$. The simulation proceeds until the carbon source (e.g., glucose) is exhausted. The result of the simulation is a set of fluxes, cell concentration and extracellular metabolite concentration for each time point (see Figure 3). The fluxes will be the base for calculating transcriptomics, proteomics, and metabolomics data, as shown below.

\section{Generating Proteomics Data}

The flux values obtained from FBA are subsequently used to generate proteomics data, which describe the concentration of the protein catalyzing a given reaction within the host organism. The protein concentration for each time point is derived from the corresponding fluxes through a linear relationship:

$$
P_{j}=V_{j} / k+\beta
$$

which is loosely inspired in the Michaelis-Menten equation (Heinrich and Schuster, 1996), where $V_{j}$ is the flux of reaction $j, P_{j}$ is the concentration of the protein catalyzing the reaction $j$, and $k$ is a linear constant arbitrarily set to 0.1 . The symbol $\beta$ is an added random noise which is set to $5 \%$ of the signal.

\section{Generating Transcriptomics Data}

The aforementioned proteomics values are subsequently used to generate transcriptomics data, which describe the abundance of RNA transcripts linked to a given protein within the host organism. For simplicity, the transcript data is assumed to have a linear relationship with the proteomics data:

$$
T_{j}=P_{j} / q+\gamma
$$

where $T_{j}$ is the abundance of RNA transcripts linked to the reaction $j$, and $q$ is a linear constant arbitrarily set to 0.833 . As above, $\gamma$ is a random noise addition set to $5 \%$ of the signal data. This calculation is performed for each time point.

\section{Generating Metabolomics Data}

The flux values obtained from the FBA are also used to generate the metabolomics data, which describe the concentration of a given metabolite within the host organism. While finding the metabolite concentrations compatible with a given metabolic flux, protein concentrations, and transcript levels is a non-trivial endeavor, here we attempt to produce metabolite profiles that are not obviously unreasonable. Hence, we want concentrations of zero for metabolites that are connected to fluxes that are null, and non-zero in any other case. The easiest way to achieve this is by averaging the absolute value of all the fluxes producing or consuming the desired metabolite:

$$
M_{i}=\Sigma_{j}\left|S_{i j} V_{j}\right| / n
$$

where $M_{i}$ is the concentration of the metabolite $i, j$ is a reaction that involves metabolite $i$, and $n$ is the total number of reactions in which metabolite $i$ participates. This calculation is performed for each time point.

\section{Generating Training Data for Machine Learning and Testing Predictions}

We leverage the OMG library to create training data to showcase the use of ART to guide bioengineering (Figure 2). We will first create multiomics (transcriptomics, proteomics, metabolomics), cell concentration, and extracellular metabolite concentration data for the wild type E. coli strain (WT). Then we will use ART to suggest initial WT modifications (designs) so as to create enough data to train ART to be predictive. Those initial designs will be used by OMG to simulate isoprenol production data for bioengineered strains that include the genetic modifications indicated by the initial designs. ART will be trained on these data, and then used to suggest strain modifications that are predicted to increase isoprenol production. We will then compare ART predictions for isoprenol production with the "observed" results produced by using those designs to simulate bioengineered strains through OMG. In sum, OMG results are used as ground truth to be leveraged in testing ART's performance.

This process involves the following phases:

1. Choosing input and response variables. Since the objective is to improve production of isoprenol, we use isoprenol concentration as the response variable. By inspecting the $E$. coli network we choose the following fluxes connected to acetyl-CoA, which is the source for the isoprenol pathway: ACCOAC, MDH, PTAr, CS, ACACT1r, PPC, PPCK, PFL. These fluxes then form the set of input variables for ART (Radivojević et al., 2020).

2. Representation of different strain designs (i.e., genetic modifications). We will consider only two types of modifications for each flux: knock-out (KO) and doubling the flux (UP). This choice results in three categories for each of the fluxes, which additionally include no modification (NoMod). We denote these categories by 0,1 , and 2 for KO, NoMod, and UP, respectively. Considering eight fluxes and three options for design of each, the total number of possible designs is $3^{8}=6,561$.

3. Choose training data size. We choose the initial training data to consist of 96 nonequivalent designs (instances), including the WT strain. This choice mimics one 96 -well plate run. Different designs (instances) here represent different engineered strains. These 96 designs represent about $1.46 \%$ of all design space.

4. Generate initial designs. Initial designs are generated using ART's feature for generating recommendations for the initial cycle, by setting its input parameter initial_cycle to True. This ART functionality relies on the Latin Hypercube method (McKay et al., 1979), which spaces out draws in a way that ensures the set of samples represents the variability of the full design space. Another parameter needed for ART is num_recommendations, which we set to 95 (see point 3 above). See the ART publication (Radivojević et al., 2020) for a list of other optional parameters. As the current version of ART deals only with continuous variables for the initial cycle, 


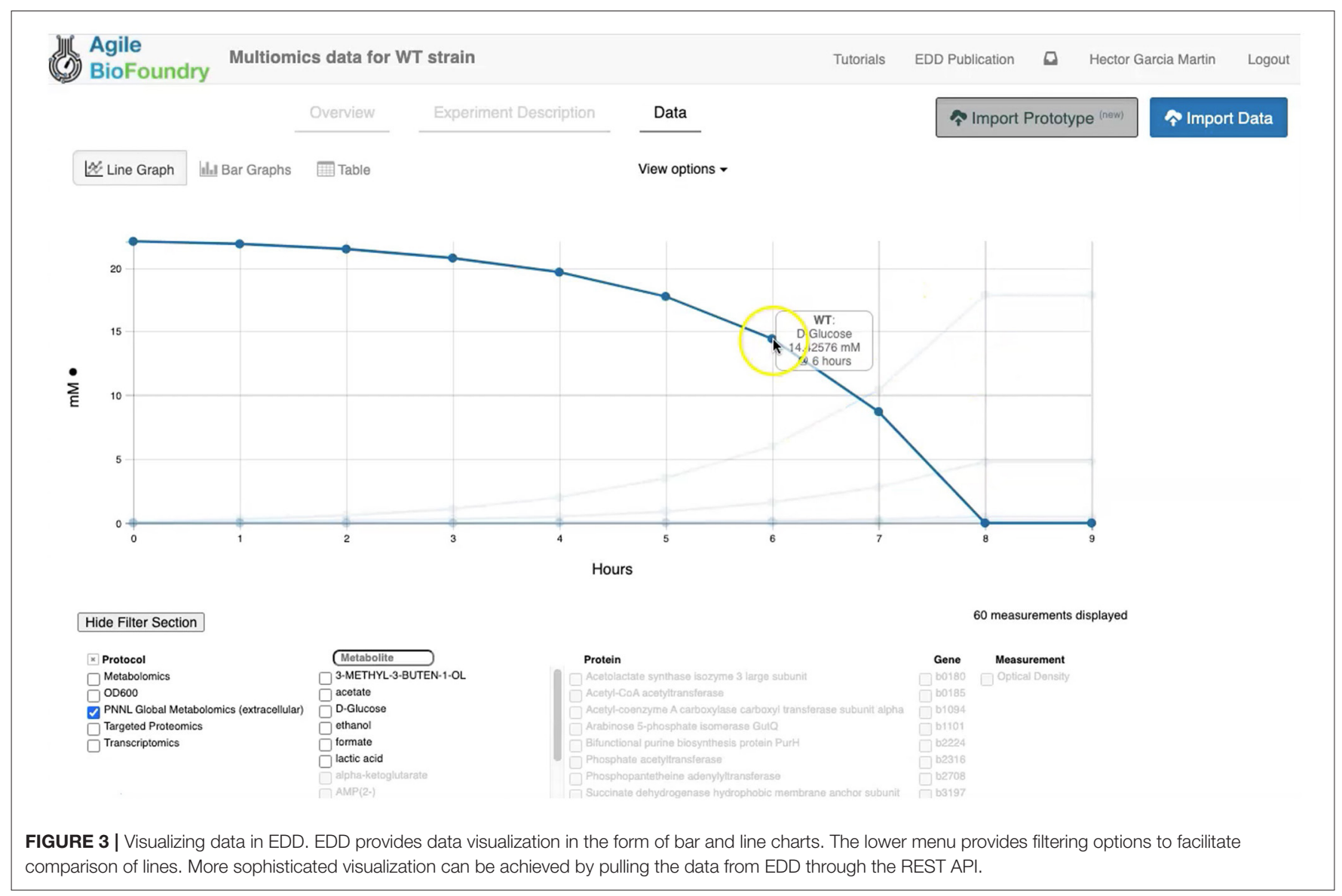

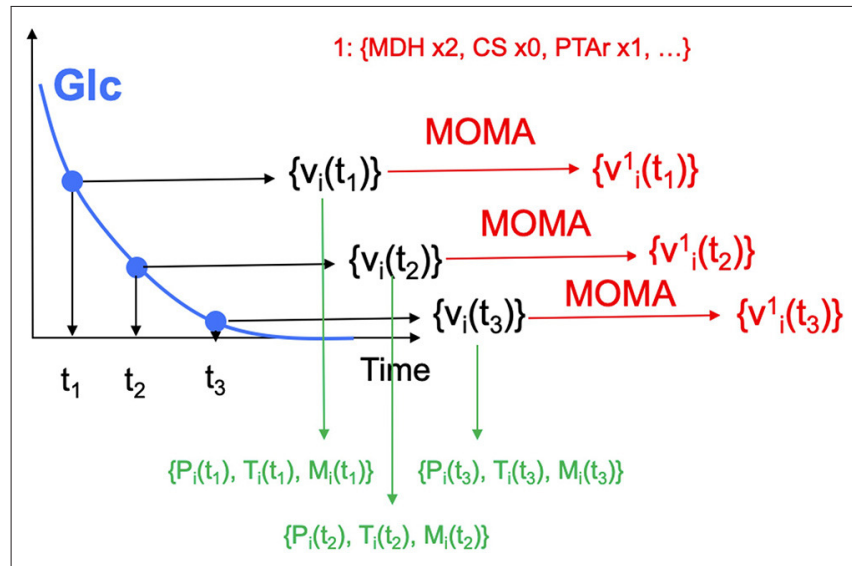

FIGURE 4 | Generating multiomics time series data. For each time point, we generate fluxes by solving an FBA problem, until glucose is fully consumed. MOMA is used in conjunction with the design (e.g., increase MDH flux 2-fold, knock CS out, maintain PTAr) to predict fluxes for the strain bioengineered according to the design.

ART's recommendations will be drawn from interval $[0,1]$, which is the default interval if no specific upper and lower bounds are provided in a separate file. We then transform each of those values into one of the defined categories $\{0,1,2\}$ by applying the function $\mathrm{f}(\mathrm{x})=3^{*}$ floor $(\mathrm{x})$. Finally, we add a WT strain design $\{1,1, \ldots, 1\}$. See Jupyter Notebook B for the details.

5. Generate production data for the initial designs. The initial designs from ART are used as input to the OMG library, generating our "ground truth" for the isoprenol production levels for each of the initial designs. This represents the strain construction and the corresponding phenotyping experiments, which are simulated through OMG's mechanistic modeling. In order to simulate how production is affected by the genetic changes suggested in the designs (e.g., knock out $\mathrm{MDH}$, upregulate PFL and do not change CS), we used MOMA (Segrè et al., 2002) for each of the time points in the flux series (Figure 4). Details can be found in Notebook C.

6. Training ART with initial production data. ART uses the initial designs and their corresponding productions from phases 4 and 5 to train, enabling it to predict isoprenol production for designs it has not seen. Details can be found in Jupyter Notebook D.

7. Generate next-cycle design recommendations using ART. Once trained, ART generates 10 recommendations that are expected to improve isoprenol production. Predictions are generated for each recommendation in the form of probability distributions (Figure 5). Details can be found in Notebook D.

8. Compare ART predictions to ground truth. Finally, we take ART's design recommendations from phase 7 


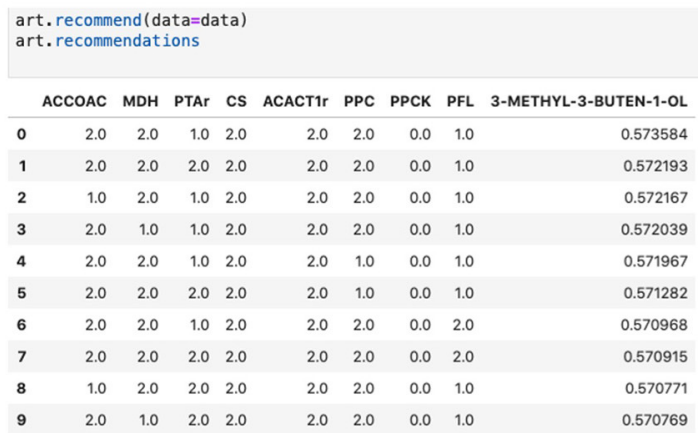

It turns out that all recommendations indicate that the CS and ACACT1r reaction fluxes should double and PPCK should be knocked out.

We can visualize the predicted distribution for all recommendations:

pred col_name $=$ 'Mean predicted Isoprenol [mM]'

plot.recomm_distribution(art, art.find_current_best(), [pred_col_name])

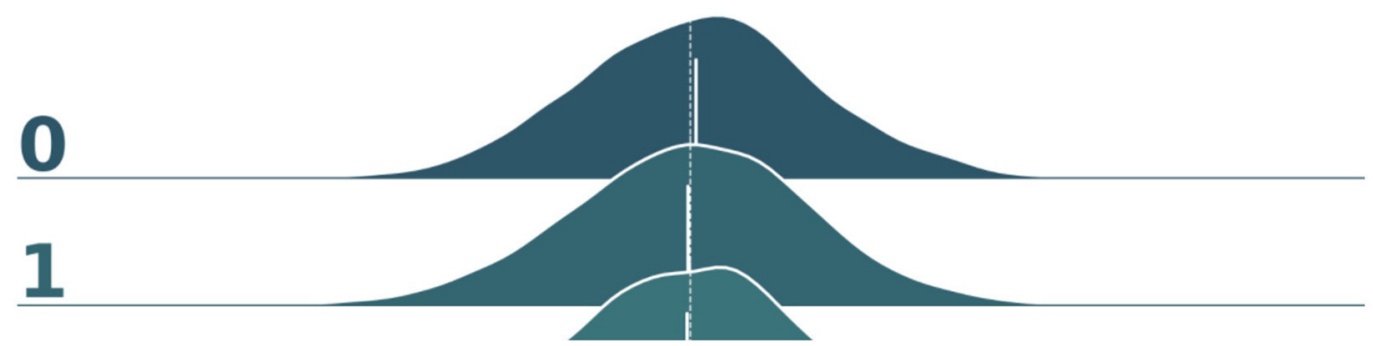

FIGURE 5 | Using machine learning to predict production and recommend new designs. The ART library takes a DataFrame containing input designs (i.e., which fluxes to overexpress, 2 , keep the same, 1 , or knock out, 0) and isoprenol production (response). The trained model recommends new designs that have the highest production. The recommendations come with predictions of production in a probabilistic fashion: i.e., the probability of production of 10, 15, 25, $40 \mathrm{mMol}$, etc.
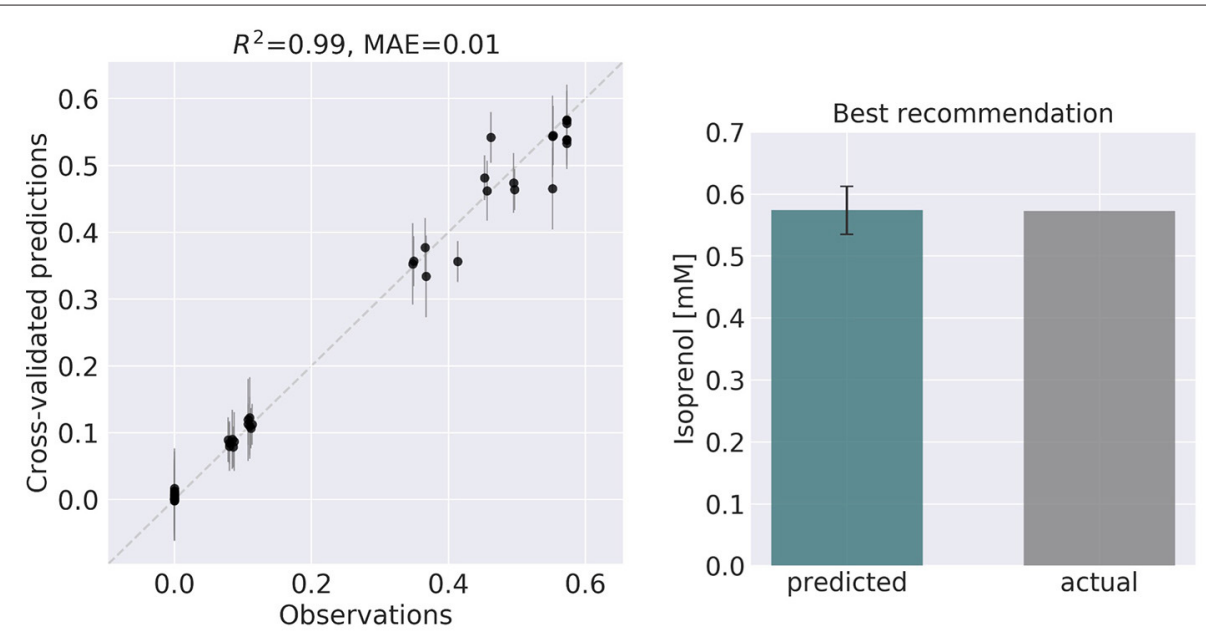

FIGURE 6 | ART recommendations display production levels production very similar to predictions. Left panel compares cross-validated predictions for isoprenol production from ART versus the values obtained through the OMG library for the training data set. Cross-validation keeps a part of the data set hidden from the training to compare against predictions, providing a good idea of the quality of predictions for new data sets. The right panel compares the predicted production for the recommended strain (\#97) vs the actual production as generated through the OMG library. The comparison indicates a very good agreement between the prediction and observation.

and use OMG to simulate the corresponding ground truth isoprenol productions, similarly as in phase 5 . We compare these isoprenol productions (observed production) vs. the machine learning recommendations from ART (Figure 6). Details can be found in Jupyter Notebook E. 


\section{Data Formats for Generic Input Files}

The data formats for the generic input files in EDD involve five columns. The first column specifies the line (e.g., WT). The second one is the measurement type identifier, which involves a standardized choice: Pubchem IDs for metabolites, UniProt IDs for proteins, and Genbank gene IDs for transcripts (e.g., CID: 715 , acetate). The third column is the time point (e.g., $0 \mathrm{~h}$ ). The fourth column is a value for the corresponding identifier (e.g., 2.4). The final column is the unit corresponding to this value: FPKM for transcriptomics data, proteins/cell for proteomics data, and $\mathrm{mg} / \mathrm{L}$ or $\mathrm{mM}$ for metabolomics data. Optical Density (OD) has no units.

\section{RESULTS AND DISCUSSION}

In the following sections, we will provide a step-by-step example of how to use this suite of tools (ICE, EDD, ART, OMG, and Jupyter notebooks) to guide metabolic engineering efforts and increase isoprenol production in $E$. coli for a synthetic data set (Figure 2, and "Methods" section). Using synthetic (simulated) data allows for a more effective demonstration, since there is no obstacle to creating time series multiomics data involving transcriptomics, proteomics, metabolomics, and fluxomics. For the purposes of this demonstration, creating such a data set using real experiments would be prohibitively expensive and limiting.

\section{Storing Strain Information in ICE}

Our first step involves storing the initial strain information in the Agile BioFoundry (ABF) instance of ICE: https://public-registry. agilebiofoundry.org/ (see Screencast 1 in Tables 1, 2). Storing strain information in ICE provides a standard way to document the design phase and make this information available for later use. ICE provides access controls so that the strains can be created as the experiment progresses, and then made public later (e.g., at publication time). We will initially store the information for the base strain (or wild type, WT). After creating an account and logging in, we click on "Create Entry" and choose "Strain." We then fill the relevant strain information (e.g., "Name," "Biosafety Level," "Description," "Sequence," etc.). Finally, we will click on "Submit" to create the strain entry. The strain is now available on the ICE instance and is assigned a part number that will be used to enter experimental data into EDD as the next step. Strain information can also be easily downloaded from ICE through the GUI (Screencast 5).

\section{Importing Data Into EDD}

Importing the multiomics data set into EDD allows for standardized data storage and retrieval. The use of the Experiment Data Depot (EDD) allows for the seamless integration of data generated from different analytical methods (e.g., mass spectroscopy, sequencing, HP-LC). EDD focuses on storing the biologically interpretable data: i.e., data that can be immediately interpreted by a biologist without requiring detailed knowledge of the analytical measurement technique (e.g., metabolite concentrations rather than GC-MS traces, or transcripts per cells rather than individual genomic sequencing reads). Furthermore, the use of standardized schemas is
TABLE 1 | Workflow for the paper and demonstrative Jupyter notebooks and screencasts.

\begin{tabular}{lll}
\hline Step & Description & Demonstration \\
\hline 1 & WT strain import into ICE & Screencast 1 \\
2 & WT multiomics data generation & Notebook A \\
3 & EDD import of WT multiomics data & Screencast 2 \\
4 & EDD visualization of WT multiomics data & Screencast 3 \\
5 & Initial designs generation by ART & Notebook B \\
6 & $\begin{array}{l}\text { Bulk ICE import of bioengineered (BE) } \\
\text { strains }\end{array}$ & Screencast 4 \\
7 & ICE export of BE strains & \\
8 & Isoprenol production data generation for & Notebook C \\
& BE strains & \\
9 & Bulk EDD import and visualization of BE & Screencast 6 \\
10 & strains production data & \\
11 & EDD export of BE production data & Screencast $7+$ Notebook D \\
12 & Using the ART frontend & Screencast 8 + Notebook D \\
13 & Comparing ART predictions with ground & Notebook E \\
& truth & \\
\hline
\end{tabular}

All screencasts and notebooks are enumerated in Tables 2, 3.

TABLE 2 | Screencasts.

\begin{tabular}{ll}
\hline Screencast & Description \\
\hline 1 & WT strain import into ICE \\
2 & EDD import of WT multiomics data \\
3 & EDD visualization of WT multiomics data \\
4 & Bulk ICE import of bioengineered (BE) strains \\
5 & ICE export of BE strains \\
6 & Bulk EDD import and visualization of BE strains production data \\
7 & EDD export of BE production data \\
8 & ART predictions and recommendations \\
9 & Using the ART frontend
\end{tabular}

fundamental for downstream analysis such as machine learning or mechanistic modeling. Indeed, it is estimated that $50-80 \%$ of a data scientist's time is spent with the type of data wrangling that EDD avoids by providing an ontology (For Big-Data Scientists, 'Janitor Work' Is Key Hurdle to Insights - The New York Times) (Lohr, 2014). This ontology reflects the objects and processes most often encountered in metabolic engineering experiments (see Figure 2 in Morrell et al., 2017). For example, a typical metabolic engineering project starts by obtaining several strains from a strain repository (e.g., DMZ, ATCC, ICE). Those strains are cultured in different flasks under different conditions (different media, induction levels etc.), which we call lines because they are a combination of strain and culture conditions, and represent a different line of enquiry or question being asked (e.g., does this strain under this condition improve production?). The final steps usually involve making measurements relevant to the experiment's ultimate goal. These measurements could be the concentration of the metabolite that the strains are engineered 


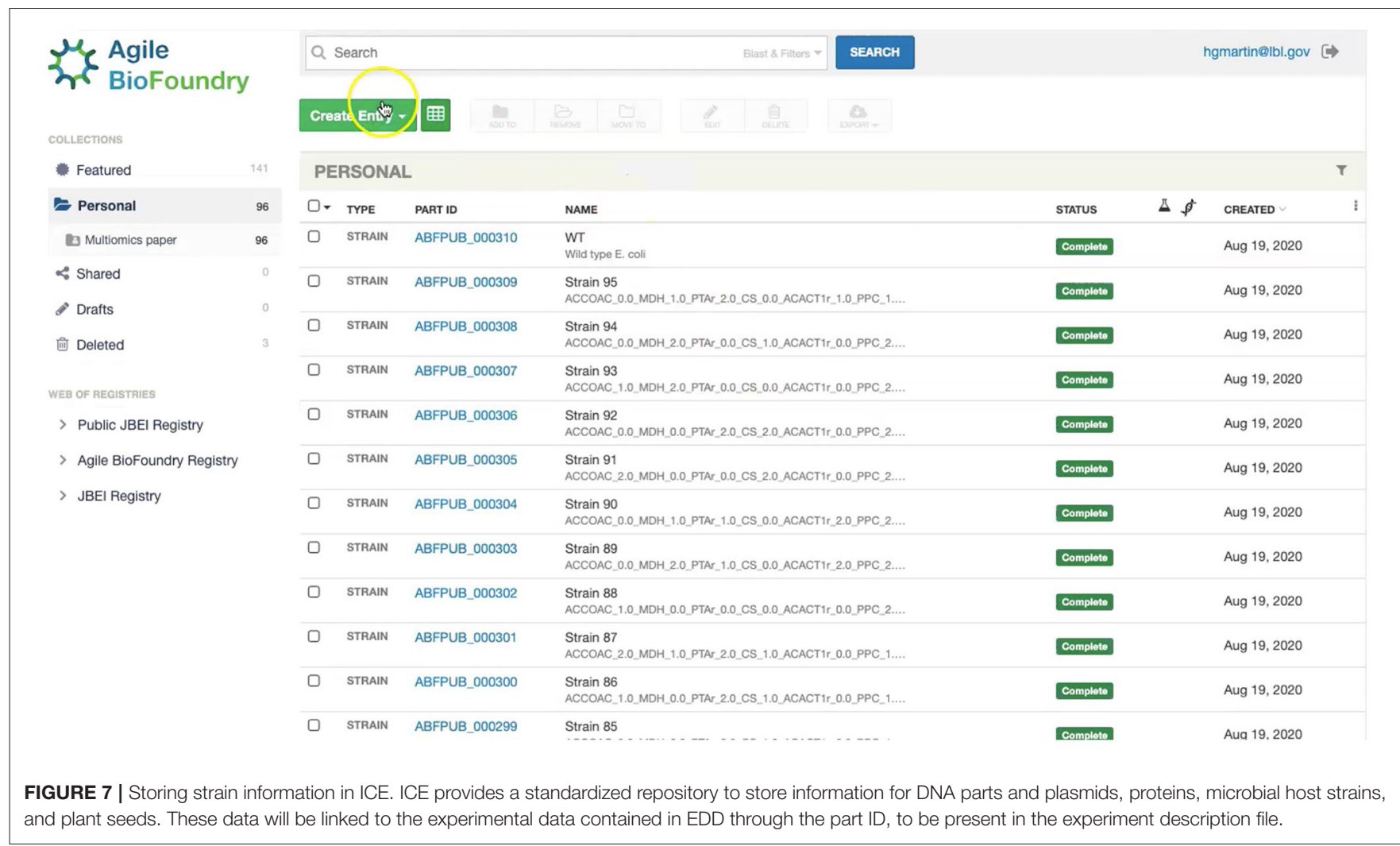

to produce (e.g., isoprenol in our example), or a transcriptomics or proteomics analysis that describes the amount of gene transcription or protein expression. Each of these measurements is obtained by applying a protocol (e.g., proteomics) to a given line, resulting in an assay. Performing an assay results in a set of measurement data: e.g., the number of grams of acetate per liter in the media, or the number of proteins per cell. In this ontology, assays can include one or more time points. As a general rule, destructive assays (e.g., proteomics through LCMS) include one time point per assay, and non-destructive assays (e.g., continuous measurement of cell optical density through an optode in a fermentation platform such as a biolector) include several time points in the same assay. All this information is collected in a study, which is used to describe a single continuous experiment (e.g., using measuring isoprenol production for all bioengineered strains).

The first step in the data import involves creating a study and uploading an "Experiment description" file, which collects all the experimental design and metadata (see Screencast 2). The "Experiment description" file describes the strains being used through a "Part ID" number tied to a strain repository, such as ICE (Figure 7). This file also contains metadata relevant to the experiment (e.g., temperature, culture shaking speed, culture volume etc.). The "Experiment description" file should not include any result data. Often, the distinction between data and metadata is crystal clear, but it can be blurred in the case of concatenated experiments: e.g., the hydrolysate sugar concentration for a plant deconstructed with ionic liquid can be data for a deconstruction experiment, but metadata for an experiment focused on the fermentation of that hydrolysate through a bioengineered strain. As a rule of thumb, metadata involves the information that is known before the experiment, and data is the information that is only obtained by performing the experiment. Once the "Experiment description" file is added, you should be able to see all the experimental design data under the "Experiment description" tab in EDD.

The second step in data input involves uploading the data files for each assay. In order to do so, click on "Import data," and you will access EDD's new streamlined import. This new import emphasizes clarity and usability, and starts by prompting for the "Data category" to be uploaded (Figure 8). Data categories involve broad umbrellas of data types such as: transcripts, proteins, metabolites, or other data. The next choice involves the actual specific protocol used for acquiring the data. There are many types of, e.g., proteomics protocols which differ in extraction protocols, as well as the type of mass spectrometer used and its setting (chromatography column, gradient time, etc.). We encourage the use of formal protocol repositories which provide DOIs (digital object identifiers), such as protocols.io (Teytelman et al., 2016), to encourage reproducibility. Protocols can be added by the system administrator in charge of the EDD instance used. The next choice involves the type of file used to input the data. All protocols include a generic file type which is the simplest possibility (see "Data Formats for Generic Input Files" section). More complex file types can be easily added through scripts that map into this generic file type. The file upload completes the 


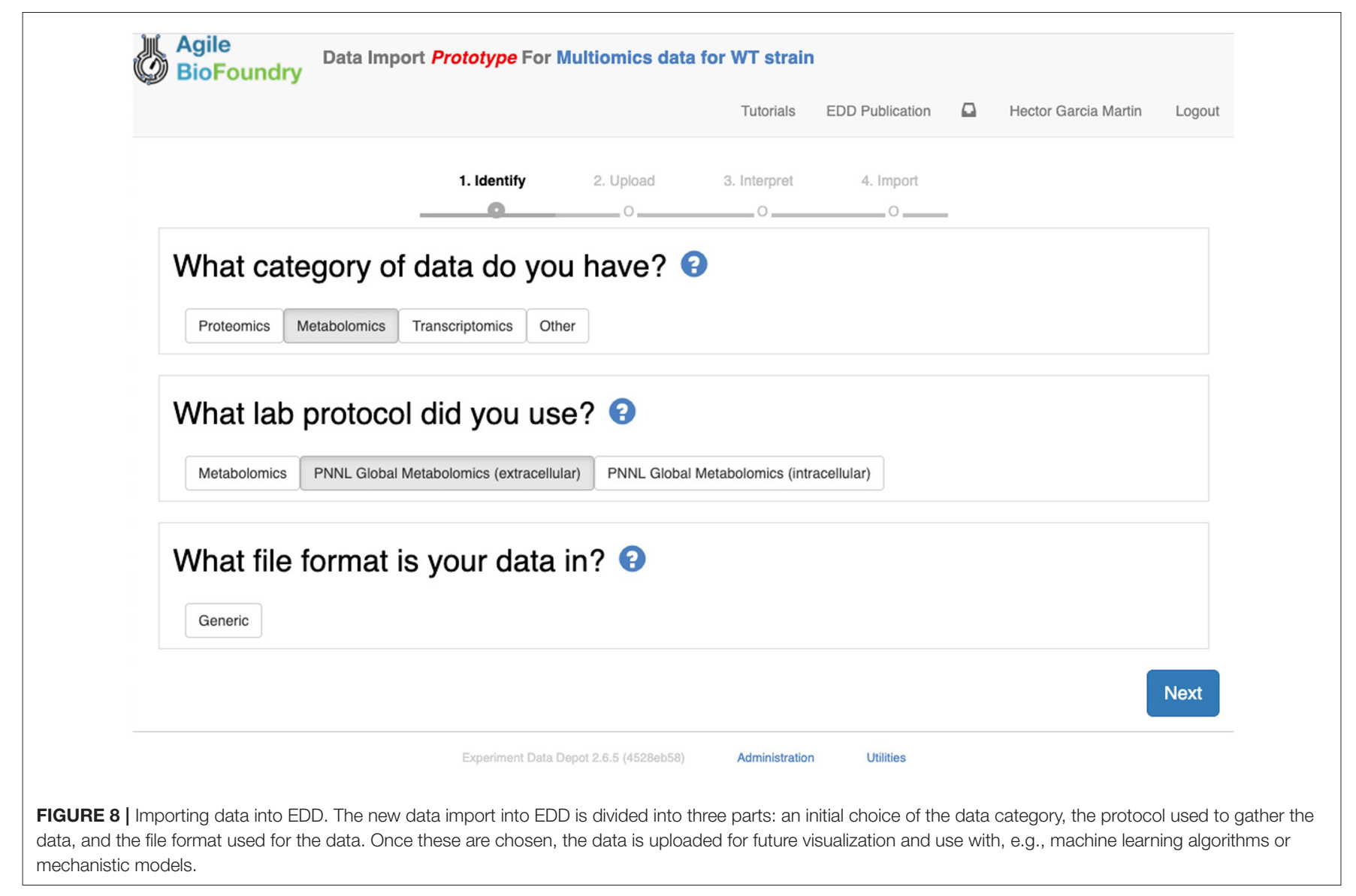

data import, and these data can be now found under the "Data" tab in the study. Similar to entering strains in ICE, data and metadata import into EDD creates incremental documentation of the experiment in a form that can easily be published later by modifying the study's access controls.

We import data into EDD twice in our metabolic engineering workflow (Figure 2): steps 3 and 9 in Table 1. In the first case (Screencast 2), we upload data created through the OMG library for the wild type (WT, Notebook A). Later (step 8) we use OMG to simulate isoprenol production data for the 95 bioengineered strains proposed by ART (Notebook C), and upload that data into EDD (step 9, Screencast 6).

\section{Visualizing Data in EDD}

EDD provides data visualization for the comparison of multiomics data sets though line and bar graphs. For example, you can easily compare the synthetic data sets created for this manuscript, which include the cell density, extracellular metabolites, transcriptomics, proteomics and metabolomics data for the base strain (Figure 2). Once in EDD, the data can be viewed by clicking on the "Data" tab in the corresponding EDD study (see Screencast 3, Figure 3). The default view is the "Line Graph" view, which displays the data as times series, with the time dimension on the $x$-axis and the measurements on the $y$-axis. Each different measurement unit is given an axis (e.g., $\mathrm{mg} / \mathrm{L}$ for metabolites, proteins/cell for proteomics, FPKM for transcriptomics). The filters at the bottom of the screen allow the users to choose the data they want to concentrate on: e.g., only transcriptomics, only proteomics, only metabolites, only metabolite "octanoate," or only proteins "Galactokinase" and "Maltoporin," or only gene "b0344" and protein "Enolase." It is also possible to view the data in the form of bar graphs clustered by measurement, line, or time. The "Table" tab shows a quick summary of all data available in the study.

EDD provides basic visualization capabilities to check data quality and compare different lines and data types. Users who want a customized visualization or figure can download the data into a pandas DataFrame (pandas - Python Data Analysis Library) (McKinney, 2015) through the REST API (see next section), and use Python to leverage any of the available visualization libraries: e.g., Matplotlib (Yim et al., 2018) or Seaborn (Seaborn: Statistical Data Visualization - Seaborn 0.10.1 Documentation) (Waskom et al., 2020).

EDD visualization is demonstrated for two very different cases in two steps of our metabolic engineering workflow (Figure 2): steps 4 (Screencast 3) and 9 (Screencast 6). The first case involves visualizing multiomics data for a single strain (WT, Figure 3), whereas the second case involves the visualization of shallow data (final isoprenol production at a final point) for 96 different strains. 


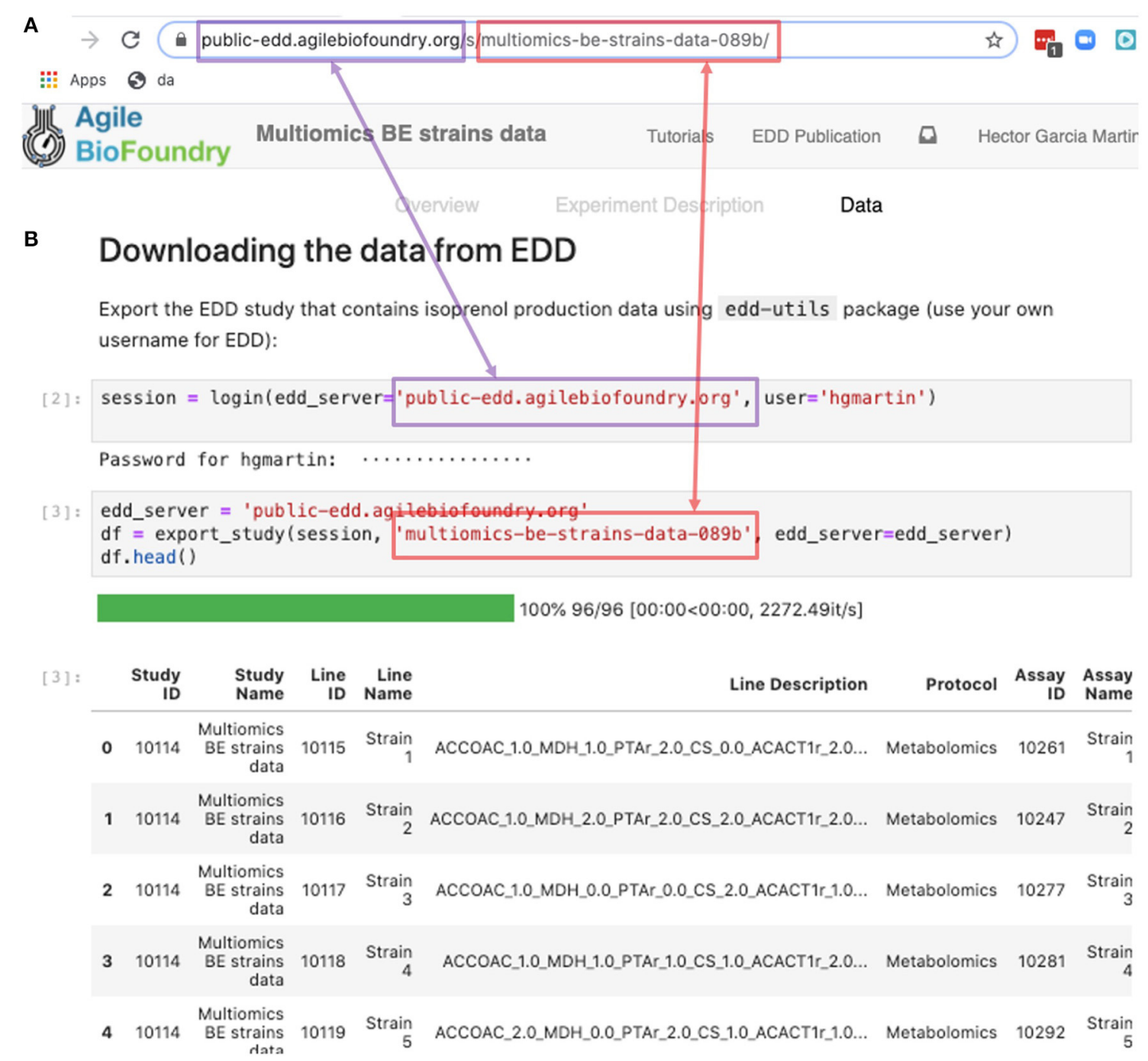

FIGURE 9 | Exporting data from EDD into an executable Jupyter notebook for downstream processing. The EDD study web address (A) provides the server (magenta) and the slug (red) to export the study data in the form of a pandas DataFrame into a Jupyter notebook (B). Once in a DataFrame format in a Jupyter notebook, a plethora of Python libraries are available for visualization, mechanistic modeling or machine learning.

\section{Exporting Data From EDD}

Data can be exported from EDD in two ways: a manual CSV file download, or a REpresentational State Transfer (REST) (Masse, 2011) Application Programming Interface (API). The REST API is the preferred method since it is easy to use, convenient, and flexible.

CSV export works through the Graphical User Interface (GUI) found in the "Table" tab. By selecting the desired measurements and clicking on "Export Data," the user can access a menu that provides options for layout and metadata to be included, as well as a visual example of the export. A CSV file is then generated by clicking on "Download."

The REST API provides a way to download the data in a form that can be easily integrated into a Jupyter notebook (see Figure 9, Screencast 7, and Table 3). A Jupyter notebook is a document that contains live code, equations, visualizations and
TABLE 3 | Jupyter notebooks.

\begin{tabular}{ll}
\hline Jupyter notebook & Description \\
\hline A & WT multiomics data generation \\
B & Initial designs generation by ART \\
C & Isoprenol production data generation for BE strains \\
D & EDD export and ART recommendations \\
E & Comparing ART predictions with ground truth
\end{tabular}

explanatory text (Project Jupyter | Home ${ }^{1}$; Jupyter Notebooks) (Kluyver et al., 2016). The edd-utils package uses EDD's REST API to provide a DataFrame inside of your Jupyter notebook to visualize and manipulate as desired. A DataFrame is a structure from the popular library Pandas (Python ANd Data 


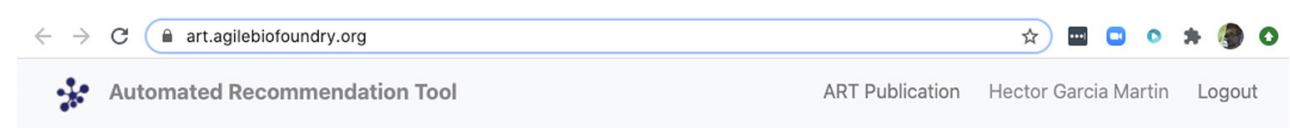

File Upload

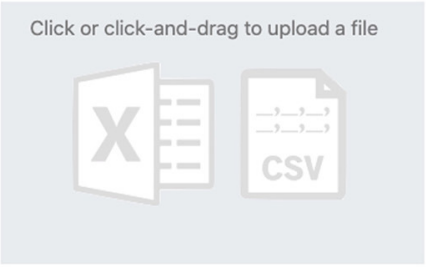

ART (1873239), Frontend (6b5c580) Administration

FIGURE 10 | ART also provides a frontend that does not require coding. The frontend can be found at https://art.agilebiofoundry.org/ and provides the main functionality of the ART library (Figure 5) in an intuitive interface. The frontend also provides a REST API that users with coding experience can leverage to use Berkeley Lab's compute resources for running ART, or to trigger ART runs automatically from other code.

AnalysiS) (pandas - Python Data Analysis Library) (McKinney, 2015), that focuses on providing tools for data analysis in python. The combination of Pandas with Jupyter notebooks provides reproducible workflows and the capability to do automated data analyses (see Screencast 8). The users can run the export_study() function from the edd-utils package (see code availability) to download a study from a particular EDD instance. The study is identified by its slug: the last part of the internet address corresponding to the EDD study (Figure 9). The EDD instance is identified by its internet address (e.g., http:// public-edd.agilebiofoundry.org/ or https://public-edd.jbei.org/). Anyone with an approved account can use the EDD instances hosted at those addresses, or anyone can create their own EDD instance by downloading and installing the open source EDD software (see code availability). EDD includes access controls, e.g., for preventing dissemination of experimental data prior to publication.

EDD export is demonstrated in a single step in our workflow (Figure 2): the export of production data for the bioengineered strains in step 10 (Screencast 7). These data will be used to train the machine learning algorithms in the Automated Recommendation Tools, and recommend new designs.

\section{Recommending New Designs Through ART}

The data stored in EDD and ICE can be used to train machine learning methods and recommend new experiments. We will now show, for example, how to use the data we uploaded into EDD to suggest how to improve the final production of isoprenol, by using the Automated Recommendation Tool (ART) (Radivojević et al., 2020). ART is a tool that combines machine learning and Bayesian inference to provide a probabilistic predictive model of production, as well as recommendations for next steps (Figure 5, see technical details in Radivojević et al., 2020). In this case, we will use as input the genetic modifications on the strain (e.g., knockout ACCOAC, overexpress $\mathrm{MDH}$, maintain CS, etc.) and we will try to predict final isoprenol production (see "Generating training data for machine learning and testing predictions" section).

Firstly, we will adapt the DataFrame obtained previously (step 10 in Table 1) to provide training data for ART. All we need to provide ART is the input (genetic modifications) and response (final isoprenol production) for each of the 96 instances. We do this by expanding the line description to include the design details into several new columns detailing the specified genetic modification for each reaction (Screencast 8, Notebook D). Next, we specify ART's parameters: input variables, response variable, number of recommendations, etc. ART uses these data and parameters to train a predictive model, which is able to recapitulate quite effectively the observed isoprenol production for the training data set (Figure 6). Recommendations for designs that are predicted to increase production are also provided by ART (Figure 5).

We can also use ART's web-based graphical frontend to produce recommendations, if we prefer not to use code (Figure 10). ART's frontend can be found at https:// art.agilebiofoundry.org/, and requires creating an account (Screencast 9). Once the account is created and approved, we can provide the same DataFrame we created above in CSV or Excel format (Notebook D). We can then select the input variables and response variables, select the objective (maximization, minimization or reach a target value), a threshold for declaring success, and then press "Start." The job is sent to the server and an email is sent to the user once it is finished.

ART's recommendations suggest that knocking out the PPCK flux and either maintaining or overexpressing all other seven fluxes should increase production of isoprenol from $0.46 \mathrm{mMol}$ to $0.57 \mathrm{mMol}$ ( $23 \%$ increase, Figure 5). The machine learning model suggests that these are the best combinations based on the predictive probability. We can check this result through the OMG library, which we consider our ground truth (Figure 2). 
Indeed, isoprenol production for this design is $0.57 \mathrm{mMol}$ vs. the predicted $0.57 \pm 0.02$ (Figure 6). Hence, ART has been able to predict which combination of designs would produce a production increase. This is a non-trivial endeavor, since only $11 \%$ of designs actually improve production, according to the synthetic data provided by OMG.

\section{CONCLUSION}

In conclusion, we have shown that the combination of tools presented here $(\mathrm{ICE}+\mathrm{EDD}+\mathrm{ART}+\mathrm{OMG}+$ Jupyter notebooks) provide a standardized manner to store data so it can be leveraged to produce actionable recommendations. We have shown how to use ICE to store strain information, EDD to store experiment data and metadata, and ART to leverage these data to suggest new experiments that improve isoprenol production. By combining these tools we have shown how to pinpoint genetic modifications that improve production of isoprenol, a potential biofuel, by $23 \%$ (from $0.46 \mathrm{mMol}$ to $0.57 \mathrm{mMol}$, Figure 5), in a simulated data set (through $\mathrm{OMG}$ ). The same procedures are applicable in the case of real experimental data. In sum, this set of tools provides a solution for the data deluge that bioengineering is currently experiencing, and a way to build on preexisting data to fruitfully direct future research.

\section{CODE AVAILABILITY}

All the code used in this paper can be found in the following github repositories:

- The Jupyter Notebooks associated with this paper, as well as the input files: https://github.com/AgileBioFoundry/ multiomicspaper

- OMG: https://github.com/JBEI/OMG

- ICE: https://github.com/JBEI/ice

- EDD: https://github.com/JBEI/EDD

- The edd-utils package: https://github.com/JBEI/edd-utils

- ART: https://github.com/JBEI/ART

\section{DATA AVAILABILITY STATEMENT}

Data are available in the multiomics github repository. See Jupyter notebooks (Table 3) for exact file location. In addition, the collection for initial designs (screencast 5) is stored in a public ICE instance at https://public-registry.agilebiofoundry.

\section{REFERENCES}

Ajikumar, P. K., Xiao, W.-H., Tyo, K. E. J., Wang, Y., Simeon, F., Leonard, E., et al. (2010). Isoprenoid pathway optimization for Taxol precursor overproduction in Escherichia coli. Science 330, 70-74. doi: 10.1126/science.1191652

Beller, H. R., Lee, T. S., and Katz, L. (2015). Natural products as biofuels and biobased chemicals: fatty acids and isoprenoids. Nat. Prod. Rep. 32, 1508-1526. doi: $10.1039 / \mathrm{C} 5 \mathrm{NP} 00068 \mathrm{H}$

Bryksin, A. V., Brown, A. C., Baksh, M. M., Finn, M. G., and Barker, T. H. (2014). Learning from nature - novel synthetic biology approaches for biomaterial design. Acta Biomater. 10, 1761-1769. doi: 10.1016/j.actbio.2014.01.019 org/folders/8. EDD entries for WT and BE strains (screencasts 2 and 6) are stored at https://public-edd.agilebiofoundry.org/ s/multiomics-data-for-wt-strain-c450/ and https://publicedd.agilebiofoundry.org/s/multiomics-be-strains-data-089b/, respectively.

\section{AUTHOR CONTRIBUTIONS}

SR, TR, JM, and HGM created and developed the concept for the paper. SR, JM, TR, and HGM developed the OMG library. TR and HGM created the Jupyter Notebooks. HGM created the screencasts. MF and WM developed the ART frontend. MF contributed error checking and bug fix code to ART. MF, NH, TR, and HGM designed and tested the ART frontend. SR, TR, MF, WM, JK, and TB tested the screencasts and notebooks. SR, TR, MF, VJ, NH, and HGM wrote the paper. All authors contributed to the article and approved the submitted version.

\section{FUNDING}

This work was part of the DOE Agile BioFoundry (http:// agilebiofoundry.org) and the DOE Joint BioEnergy Institute (http://www.jbei.org), supported by the U. S. Department of Energy, Energy Efficiency and Renewable Energy, Bioenergy Technologies Office, and the Office of Science, through contract DE-AC02-05CH11231 between Lawrence Berkeley National Laboratory and the U.S. Department of Energy. This research is also supported by the Basque Government through the BERC 20182021 program and by Spanish Ministry of Economy and Competitiveness MINECO: BCAM Severo Ochoa excellence accreditation SEV-2017-0718.

\section{ACKNOWLEDGMENTS}

The authors thank Zak Costello and Jacob Coble for their contributions during the initial development of the ART frontend.

\section{SUPPLEMENTARY MATERIAL}

The Supplementary Material for this article can be found online at: https://www.frontiersin.org/articles/10.3389/fbioe. 2021.612893/full\#supplementary-material

Canton, B., Labno, A., and Endy, D. (2008). Refinement and standardization of synthetic biological parts and devices. Nat. Biotechnol. 26, 787-793. doi: $10.1038 /$ nbt1413

Carbonell, P., Radivojevic, T., and García Martín, H. (2019). Opportunities at the intersection of synthetic biology, machine learning, and automation. ACS Synth. Biol. 8, 1474-1477. doi: 10.1021/acssynbio.8b00540

Chen, Y., Guenther, J. M., Gin, J. W., Chan, L. J. G., Costello, Z., Ogorzalek, T. L., et al. (2019). Automated "cells-to-peptides" sample preparation workflow for high-throughput, quantitative proteomic assays of microbes. J. Proteome Res. 18, 3752-3761. doi: 10.1021/acs.jproteome. $9 \mathrm{~b} 00455$ 
Chubukov, V., Mukhopadhyay, A., Petzold, C. J., Keasling, J. D., and Martín, H. G. (2016). Synthetic and systems biology for microbial production of commodity chemicals. NPJ Syst. Biol. Appl. 2:16009. doi: 10.1038/npjsba.2016.9

Doudna, J. A., and Charpentier, E. (2014). Genome editing. The new frontier of genome engineering with CRISPR-Cas9. Science 346:1258096. doi: $10.1126 /$ science. 1258096

Ebrahim, A., Lerman, J. A., Palsson, B. O., and Hyduke, D. R. (2013). COBRApy: constraints-based reconstruction and analysis for python. BMC Syst. Biol. 7:74. doi: 10.1186/1752-0509-7-74

Fuhrer, T., and Zamboni, N. (2015). High-throughput discovery metabolomics. Curr. Opin. Biotechnol. 31, 73-78. doi: 10.1016/j.copbio.2014.08.006

Gardner, T. S. (2013). Synthetic biology: from hype to impact. Trends Biotechnol. 31, 123-125. doi: 10.1016/j.tibtech.2013.01.018

Ham, T. S., Dmytriv, Z., Plahar, H., Chen, J., Hillson, N. J., and Keasling, J. D. (2012). Design, implementation and practice of JBEI-ICE: an open source biological part registry platform and tools. Nucleic Acids Res. 40:e141. doi: $10.1093 /$ nar/gks531

Heinrich, R., and Schuster, S. (1996). The Regulation of Cellular Systems. Boston, MA: Springer US.

Hodgman, C. E., and Jewett, M. C. (2012). Cell-free synthetic biology: thinking outside the cell. Metab. Eng. 14, 261-269. doi: 10.1016/j.ymben.2011.09.002

Kang, A., Mendez-Perez, D., Goh, E.-B., Baidoo, E. E. K., Benites, V. T., Beller, H. R., et al. (2019). Optimization of the IPP-bypass mevalonate pathway and fedbatch fermentation for the production of isoprenol in Escherichia coli. Metab. Eng. 56, 85-96. doi: 10.1016/j.ymben.2019.09.003

Kluyver, T., Ragan-Kelley, B., Pérez, F., Granger, B., Bussonnier, M., Frederic, J., et al. (2016). Jupyter Notebooks - A Publishing Format for Reproducible Computational Workflows. IOS Press. Available online at: http://ebooks. iospress.nl/publication/42900 (accessed August 6, 2020).

Kyrou, K., Hammond, A. M., Galizi, R., Kranjc, N., Burt, A., Beaghton, A. K., et al. (2018). A CRISPR-Cas9 gene drive targeting doublesex causes complete population suppression in caged Anopheles gambiae mosquitoes. Nat. Biotechnol. 36, 1062-1066. doi: 10.1038/nbt.4245

Lewis, N. E., Nagarajan, H., and Palsson, B. O. (2012). Constraining the metabolic genotype-phenotype relationship using a phylogeny of in silico methods. Nat. Rev. Microbiol. 10, 291-305. doi: 10.1038/nrmicro2737

Lohr, S. (2014). For big-data scientists, 'janitor work' is key hurdle to insights. New York Times 17:B4.

Ma, S., Tang, N., and Tian, J. (2012). DNA synthesis, assembly and applications in synthetic biology. Curr. Opin. Chem. Biol. 16, 260-267. doi: 10.1016/j.cbpa.2012.05.001

Masse, M. (2011). REST API Design Rulebook: Designing Consistent RESTful Web Service Interfaces. Cambridge: O'Reilly Media, Inc.

McKay, M. D., Beckman, R. J., and Conover, W. J. (1979). Comparison of three methods for selecting values of input variables in the analysis of output from a computer code. Technometrics 21, 239-245. doi: 10.1080/00401706.1979.10489755

McKinney, W. (2015). Pandas, Python Data Analysis Library. Available online at: http://pandas.pydata.org (accessed September 3, 2020).

Meat-free outsells beef (2019). Meat-free outsells beef. Nat. Biotechnol. 37, 1250-1250. doi: 10.1038/s41587-019-0313-x

Morrell, W. C., Birkel, G. W., Forrer, M., Lopez, T., Backman, T. W. H., Dussault, M., et al. (2017). The experiment data depot: a web-based software tool for biological experimental data storage, sharing, and visualization. ACS Synth. Biol. 6, 2248-2259. doi: 10.1021/acssynbio.7b00204

Müller, K. M., and Arndt, K. M. (2012). Standardization in synthetic biology. Methods Mol. Biol. 813, 23-43. doi: 10.1007/978-1-61779412-4_2

Nielsen, J., and Keasling, J. D. (2016). Engineering cellular metabolism. Cell 164, 1185-1197. doi: 10.1016/j.cell.2016.02.004

Orth, J. D., Thiele, I., and Palsson, B. Ø. (2010). What is flux balance analysis? Nat. Biotechnol. 28, 245-248. doi: 10.1038/nbt.1614

Paddon, C. J., and Keasling, J. D. (2014). Semi-synthetic artemisinin: a model for the use of synthetic biology in pharmaceutical development. Nat. Rev. Microbiol. 12, 355-367. doi: 10.1038/nrmicro3240
Peralta-Yahya, P. P., Zhang, F., del Cardayre, S. B., and Keasling, J. D. (2012). Microbial engineering for the production of advanced biofuels. Nature 488, 320-328. doi: 10.1038/nature11478

Petzold, C. J., Chan, L. J. G., Nhan, M., and Adams, P. D. (2015). Analytics for metabolic engineering. Front. Bioeng. Biotechnol. 3:135. doi: 10.3389/fbioe.2015.00135

Radivojević, T., Costello, Z., Workman, K., and Garcia Martin, H. (2020). A machine learning Automated Recommendation Tool for synthetic biology. Nat. Commun. 11:4879. doi: 10.1038/s41467-020-18008-4

Roell, M.-S., and Zurbriggen, M. D. (2020). The impact of synthetic biology for future agriculture and nutrition. Curr. Opin. Biotechnol. 61, 102-109. doi: 10.1016/j.copbio.2019.10.004

Segrè, D., Vitkup, D., and Church, G. M. (2002). Analysis of optimality in natural and perturbed metabolic networks. Proc. Natl. Acad. Sci. U.S.A. 99, 15112-15117. doi: 10.1073/pnas.232349399

Stephens, Z. D., Lee, S. Y., Faghri, F., Campbell, R. H., Zhai, C., Efron, M. J., et al. (2015). Big data: astronomical or genomical? PLoS Biol. 13:e1002195. doi: 10.1371/journal.pbio.1002195

Teytelman, L., Stoliartchouk, A., Kindler, L., and Hurwitz, B. L. (2016). Protocols.io: virtual communities for protocol development and discussion. PLoS Biol. 14:e1002538. doi: 10.1371/journal.pbio.1002538

Thiele, I., and Palsson, B. Ø. (2010). A protocol for generating a highquality genome-scale metabolic reconstruction. Nat. Protoc. 5, 93-121. doi: $10.1038 /$ nprot.2009.203

Waskom, M., Gelbart, M., Botvinnik, O., Ostblom, J., Hobson, P., Lukauskas, S., et al. (2020). mwaskom/seaborn: v0.11.1 (December 2020) [Computer software]. Zenodo. doi: 10.5281/ZENODO.592845

Yim, A., Chung, C., and Yu, A. (2018). Matplotlib for Python Developers: Effective Techniques for Data Visualization With Python. Birmingham, AL: Packt Publishing Ltd.

Zhang, J., Petersen, S. D., Radivojevic, T., Ramirez, A., Pérez-Manríquez, A., Abeliuk, E., et al. (2020). Combining mechanistic and machine learning models for predictive engineering and optimization of tryptophan metabolism. Nat. Commun. 11:4880. doi: 10.1038/s41467-020-1 7910-1

Disclaimer: The views and opinions of the authors expressed herein do not necessarily state or reflect those of the United States Government or any agency thereof. Neither the United States Government nor any agency thereof, nor any of their employees, makes any warranty, expressed or implied, or assumes any legal liability or responsibility for the accuracy, completeness, or usefulness of any information, apparatus, product, or process disclosed, or represents that its use would not infringe privately owned rights. The United States Government retains and the publisher, by accepting the article for publication, acknowledges that the United States Government retains a non-exclusive, paid-up, irrevocable, worldwide license to publish or reproduce the published form of this manuscript, or allow others to do so, for United States Government purposes. The Department of Energy will provide public access to these results of federally sponsored research in accordance with the DOE Public Access Plan (http://energy.gov/downloads/ doe-public-access-plan).

Conflict of Interest: NH declares financial interests in TeselaGen Biotechnologies, and Ansa Biotechnologies.

The remaining authors declare that the research was conducted in the absence of any commercial or financial relationships that could be construed as a potential conflict of interest.

Copyright $\odot 2021$ Roy, Radivojevic, Forrer, Marti, Jonnalagadda, Backman, Morrell, Plahar, Kim, Hillson and Garcia Martin. This is an open-access article distributed under the terms of the Creative Commons Attribution License (CC BY). The use, distribution or reproduction in other forums is permitted, provided the original author(s) and the copyright owner(s) are credited and that the original publication in this journal is cited, in accordance with accepted academic practice. No use, distribution or reproduction is permitted which does not comply with these terms. 